\title{
EDITORIAL
}

\section{CMV immunology}

\section{Guest Editor Stipan Jonjic}

Cellular \& Molecular Immunology (2015) 12, 125-127; doi:10.1038/cmi.2014.132; published online 12 January 2015

Human cytomegalovirus (HCMV) is a ubiquitous pathogen linked to a high risk of morbidity in immunologically suppressed and immunodeficient patients, and it is one of the leading viral causes of congenital infections. In addition, HCMV infections have been implicated in several chronic diseases. ${ }^{1}$ So far, strategies for coping with the consequences of HCMV infection are limited because of an incomplete understanding of viral pathogenesis. After primary infection, the immune response terminates the replication of the virus, but the virus persists in infected hosts for a lifetime and is able to reactivate despite the presence of significant preexisting immunity. The major obstacle to HCMV research is the virus's strict species specificity, which limits the use of experimental animals in HCMV research. Thus, research on animal cytomegaloviruses (CMVs) is essential for gaining insight into HCMV pathogenesis and immune surveillance. Among the animal CMVs, murine CMV (MCMV) is the most widely used model because it shares many biological, genetic and pathological properties with $\mathrm{HCMV}^{2}$

In this special issue of Cellular \& Molecular Immunology, several articles are included that discuss recent developments in CMV immunobiology, with an

Faculty of Medicine University of Rijeka, Department of Histology and Embryology and Center for Proteomics, Rijeka, Croatia Correspondence: S Jonjic, Faculty of Medicine University of Rijeka, Department of Histology and Embryology and Center for Proteomics, Brace Branchetta 20, 51000 Rijeka, Croatia.

E-mail: stipan.jonjic@medri.uniri.hr

Received: 8 December 2014; Revised: 8

December 2014; Accepted: 8 December 2014 emphasis on the viral manipulation of the immune response during primary and latent infections, the pathogenesis of congenital infections and novel strategies for intervention approaches, including CMV vaccines.

The hallmark of all CMVs is the presence of a large number of immunoevasion genes that target the immune response mechanism of the host. ${ }^{3,4}$ Although the number of immunoevasion genes underscores their significance to the immunobiology of the viral infection, we still do not understand their overall importance for the virus-host interaction and whether they benefit only the virus or whether some of them are also useful to the hos$\mathrm{t}$ (for example, in limiting immunopathological reactions). Notably, despite numerous immunoevasive mechanisms of CMV during primary infection, this virus induces potent immune responses that are able to contain its spread and establish latency. In contrast with our understanding of the role of viral immunoevasins during lytic infection, little is known about the role of CMV immune evasion during latency. Current data show that HCMV manipulates the immune response during latency and has profound effects on survival and the preservation of the latent viral genome. ${ }^{5}$ In this special issue, Wills et al. have summarized strategies for immune evasion during latent HCMV infection and have discussed how the understanding of these mechanisms could help in designing new approaches for targeting latent HCMV. ${ }^{6}$ The ultimate aim of these approaches is to prevent reactivation and life-threatening disease in some patient groups.

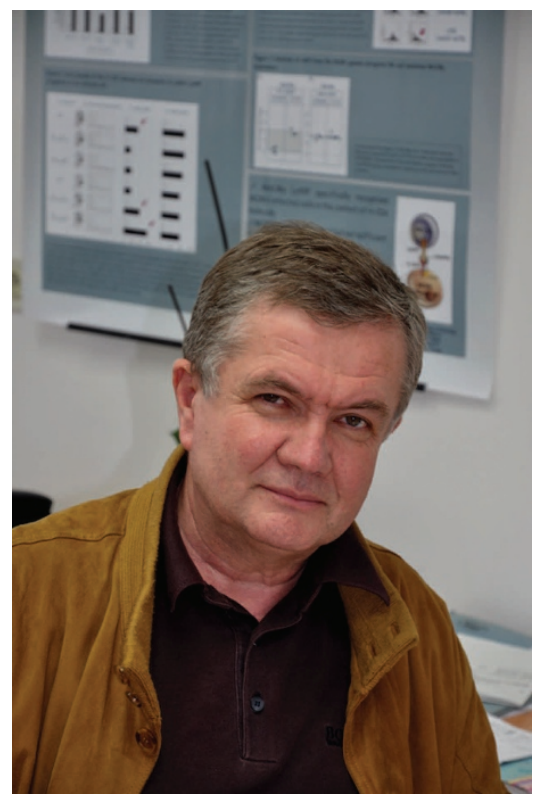

$\mathrm{CD}^{+} \mathrm{T}$ cells play an essential role in the control of CMV infection. Their protective capacity is associated with the emergence of epitope-specific effector memory $\mathrm{T}$ cells, which provide long-term protection and apparently undergo continuous activation and proliferation. These virus-specific $\mathrm{CD}^{+} \mathrm{T}$ cells have been shown to slowly accumulate after primary infection in a process known as CD8 inflation. ${ }^{7-9}$ In their review article, Halenius et al. ${ }^{10}$ have extensively reviewed the immune evasion strategies used by HCMV to manipulate both classical and non-classical MHC I molecules. By doing so, this virus manipulates the functions of $\mathrm{CD}^{+} \mathrm{T}$ cells and NK cells. The authors have also highlighted the questions that still remain to be addressed, thus providing a direction for future work. In addition, Lucin et al. ${ }^{11}$ have reviewed the 
endocytic trafficking of immune recognition molecules during CMV infection, emphasizing the mechanisms by which this virus affects the endosomal system and modulates endocytic trafficking. ${ }^{11}$

CMVs are a continuous source of surprise with respect to the mechanisms that they use to manipulate host cellular functions. In this regard, Becker et al. ${ }^{12,13}$ have presented some original studies, in which they have expanded upon their own previously published data, revealing an as-yet unknown role of mast cells in the early immune response to MCMV infection, particularly in the recruitment of CMV-specific $\mathrm{CD} 8^{+} \mathrm{T}$ cells to the site of infection. ${ }^{12,13}$ This group has characterized two waves of mast cell degranulation upon CMV infection, occurring at early and later times after infection. They have provided evidence that the first wave of mast cell degranulation is dependent on the TLR3/TRIF signaling pathway and that the second wave is caused by the infections of these cells, which occur independent of TLR3/ TRIF signaling.

An in-depth understanding of the immune responses to various pathogens is a prerequisite for designing new antiviral strategies, including vaccines. It is worth mentioning that the immune response induced by natural infection frequently does not provide sufficient protection against re-infection and disease with the same pathogen. Therefore, we need approaches that are able to induce a level of protective immunity that is superior to that induced by natural infection. CMV belongs to this group of viruses because, at least in some settings, such as congenital infection, maternal pre-existing immunity does not prevent fetal infection. The review article by McCormick and Mocarski ${ }^{14}$ focuses on HCMV vaccine strategies and immune mechanisms that determine the success of a vaccine. Much attention has been paid to the immune mechanisms governing the transplacental transmission of HCMV during pregnancy and the importance of recurrent infection in the pathogenesis of congenital HCMV diseases. The authors have emphasized that not only vaccines for $\mathrm{HCMV}$-seronegative individuals but also approaches to boost immunity in CMV-seropositive individuals are needed. In this respect, studies of mouse models have contributed a great deal to our understanding of the pathogenesis of congenital CMV infection. Newborn mice are developmentally similar to late second trimester human fetuses, and MCMV infection delivered by intraperitoneal inoculation to these mice leads to central nervous system infection, the pathogenesis of which resembles that in the HCMV-infected human fetus. Slavuljica et al. ${ }^{15}$ have discussed the major findings in the MCMV model and have summarized many aspects of congenital HCMV infection of the central nervous system, including developmental abnormalities, inflammatory lesions and functional alterations.

In summary, the significance of CMV research lies both in the advancement of our basic knowledge of viral infection and in the possibility of designing new and more efficient anti-viral treatments, including CMV vaccines. There is still a need for a deeper understanding of the immunological determinants that predispose individuals to infection because the clinical expression of HCMV infection is clearly correlated with the immune response of the host. To design more efficient prophylactic and therapeutic vaccines, it is necessary to manipulate the viral immunoevasion of the innate and adaptive immune responses to fine-tune them so that more efficient antiviral antibodies and/or more efficient T-cell responses will be generated, as has recently been shown for MCMV expressing the cellular ligand for NKG2D receptor. ${ }^{16}$ Special attention should be paid to the mechanisms of congenital HCMV infection. More efficient immune intervention approaches (such as vaccines) are needed to control the virus at the maternal-fetal interface because it is now clear that preexisting maternal immunity is insufficient for preventing infection and disease in the fetus. In summary, it can be concluded that experimental animal models will continue to be an essential source of information about CMV pathogenesis in the future.

\section{ACKNOWLEDGEMENTS}

Stipan Jonjic was supported by the EU ERC Advanced Grant and NIH R01 AI083201-01.

1 Boppana SB, Britt WJ. Synopsis of clinical aspects of human cytomegalovirus disease. In: Reddehase MJ, editor. Cytomegaloviruses: From Molecular Pathogenesis to Intervention. Norfolk: Caister Academic Press, 2013: 1-26.

2 Brune W, Hengel H, Koszinowski UH. A mouse model for cytomegalovirus infection. In: Current Protocols in Immunology. New York: John Wiley \& Sons, 1999: 19.7.1-19.7.13.

3 Jackson SE, Mason GM, Wills MR. Human cytomegalovirus immunity and immune evasion. Virus Res 2011; 157: 151-160.

4 Reddehase MJ. Antigens and immunoevasins: opponents in cytomegalovirus immune surveillance. Nat Rev Immunol 2002; 2: 831-844.

5 Mason GM, Jackson S, Okecha G, Poole E, Sissons JG, Sinclair J et al. Human cytomegalovirus latency-associated proteins elicit immune-suppressive IL-10 producing $\mathrm{CD}^{+}{ }^{+} \mathrm{T}$ cells. PLoS Pathog 2013; 9: e1003635.

6 Wills MR, Poole E, Lau B, Krishna B, Sinclair JH. The immunology of human cytomegalovirus latency: could latent infection be cleared by novel immunotherapeutic strategies? Cell Mol Immunol 2015; 12: $128-138$.

7 Karrer U, Sierro S, Wagner M, Oxenius A, Hengel $\mathrm{H}$, Koszinowski UH et al. Memory inflation: continuous accumulation of antiviral $\mathrm{CD}^{+}{ }^{+} \mathrm{T}$ cells over time. $J$ Immunol 2003; 170: 2022-2029.

8 Holtappels R, Pahl-Seibert MF, Thomas D, Reddehase MJ. Enrichment of immediateearly 1 ( $m 123 / p p 89$ ) peptide-specific CD8 $\mathrm{T}$ cells in a pulmonary CD62 $\mathrm{L}^{10}$ memoryeffector cell pool during latent murine cytomegalovirus infection of the lungs. $J$ Virol 2000; 74: 11495-11503.

9 Waller EC, Day E, Patrick Sissons JG, Wills MR. Dynamics of T cell memory in human cytomegalovirus infection. Med Microbiol Immunol 2008; 197: 83-96.

10 Halenius A, Gerke C, Hengel H. Classical and non-classical MHC I molecule manipulation by human cytomegalovirus: so many targets-but how many arrows in the quiver? Cell Mol Immunol 2015; 12 : 139-153.

11 Lucin P, Mahmutefendić H, Zagorac GB, Tomaš MI. Cytomegalovirus immune evasion by perturbation of endosomal trafficking. Cell Mol Immunol 2015; 12 : 154-169.

12 Ebert S, Becker M, Lemmermann NA, Büttner JK, Michel A, Taube $C$ et al. Mast cells expedite control of pulmonary murine cytomegalovirus infection by enhancing the recruitment of protective CD8 T cells to the lungs. PLoS Pathog 2014; 10: e1004100. 
13 Becker M, Lemmermann NA, Ebert S, Baars P, Renzaho A, Podlech J et al. Mast cells as rapid innate sensors of cytomegalovirus by TLR3/TRIF signaling-dependent and independent mechanisms. Cell Mol Immunol 2015; 12: 192-201.

14 McCormick AL, Mocarski ES. Immunologic underpinnings of vaccination to prevent cytomegalovirus disease. Cell Mol Immunol 2015; 12: 170-179.

15 Slavuljica I, Kveštak D, Huszthy PC, Kosmac $\mathrm{K}$, Britt WJ, Jonjić S. Immunobiology of congenital cytomegalovirus infection of the central nervous system - the murine cytomegalovirus model. Cell Mol Immunol 2015; 12: 180-191.
16 Trsan T, Buscheb A, Abramc M, Wensveena FM, Lemmermannd NA, Arapović M et al. Superior induction and maintenance of protective CD8 T cells in mice infected with mouse cytomegalovirus vector expressing RAE-1gamma. Proc Nat Acad Sci USA 2013; 110: 1655016555. 